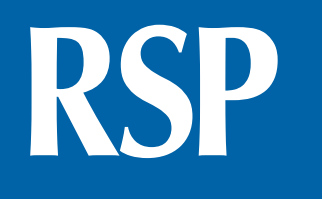

http://www.rsp.fsp.usp.br/
Revista de Saúde Pública

\title{
Qualidade de vida em indivíduos iniciando a terapia antirretroviral: um estudo de coorte
}

\author{
Gabriela Sales Pimentel' (iD, Maria das Graças Braga Ceccato"I iD, Juliana de Oliveira Costa ${ }^{\text {III,IV }}$ iD, \\ Jullye Campos Mendes' (iD, Palmira de Fátima Bonolov ${ }^{v}$ iD, Micheline Rosa Silveira" iD \\ ' Universidade Federal de Minas Gerais. Faculdade de Farmácia. Programa de Pós-Graduação em Medicamentos \\ e Assistência Farmacêutica. Belo Horizonte, MG, Brasil \\ " Universidade Federal de Minas Gerais. Faculdade de Farmácia. Departamento de Farmácia Social. Belo \\ Horizonte, MG, Brasil \\ III University of New South Wales. Centre for Big Data Research in Health. Faculty of Medicine. Sydney, Australia \\ iv Universidade Federal de Minas Gerais. Faculdade de Medicina. Pós-Graduação em Saúde Pública. Belo \\ Horizonte, MG, Brasil \\ $\checkmark$ Universidade Federal de Minas Gerais. Faculdade de Medicina. Departamento de Medicina Preventiva e \\ Social. Belo Horizonte, MG, Brasil
}

\author{
Correspondência: \\ Gabriela Sales Pimentel \\ Faculdade de Farmácia da UFMG \\ Av. Presidente Antônio Carlos, \\ 6627 , sala 1023 B2 \\ 31270-901 Belo Horizonte, MG \\ Brasil \\ E-mail: gabisalespimentel09@ \\ hotmail.com
}

Recebido: 11 jul 2020

Aprovado: 6 fev 2020

Como citar: Pimentel GS, Ceccato MGB, Costa JO, Mendes JC, Bonolo PF, Silveira MR. Qualidade de vida em indivíduos iniciando a terapia antirretroviral: um estudo de coorte. Rev Saude Publica. 2020;54:146.

Copyright: Este é um artigo de acesso aberto distribuído sob os termos da Licença de Atribuição Creative Commons, que permite uso irrestrito, distribuição e reprodução em qualquer meio, desde que o autor e a fonte originais sejam creditados.

\section{RESUMO}

OBJETIVO: Avaliar longitudinalmente a alteração da qualidade de vida em pessoas que vivem com HIV iniciando a terapia antirretroviral, atendidas em três serviços públicos de referência na assistência especializada ao HIV em Belo Horizonte.

MÉTODOS: Estudo de coorte prospectivo, com o acompanhamento de pessoas que vivem com HIV, com 18 anos de idade ou mais, e iniciando terapia antirretroviral. Dados sociodemográficos, comportamentais, clínicos, relacionados ao tratamento farmacológico e ao serviço foram obtidos por entrevistas, complementados com informações dos prontuários clínicos e dos sistemas de informação do Programa Brasileiro de HIV/AIDS. A qualidade de vida foi avaliada utilizando o instrumento WHOQOLHIV-bref, por meio de entrevista face a face, com intervalo mínimo de seis meses entre as entrevistas. A alteração média na qualidade de vida entre as duas entrevistas foi avaliada utilizando o teste $t$ pareado. Os fatores associados foram avaliados por meio de regressão linear múltipla.

RESULTADOS: A qualidade de vida global, assim como a qualidade de vida nos domínios físico, psicológico, nível de independência, ambiente e espiritual foram estatisticamente melhores em pessoas que vivem com HIV usando terapia antirretroviral no final do tempo de acompanhamento. Fatores independentemente associados ao incremento na qualidade de vida foram possuir crença religiosa e morar com outras pessoas. Enquanto ter sinais ou sintomas de ansiedade e depressão e o número de reações adversas a medicamentos reportadas foram preditores associados à piora da qualidade de vida.

CONCLUSÕES: Os resultados evidenciam melhora na qualidade de vida em pessoas vivendo com HIV iniciando a terapia antirretroviral ao longo do tempo. Evidenciam ainda a necessidade de se acompanhar e prover cuidados de saúde, em especial para indivíduos com sinais e sintomas de ansiedade e depressão e que relatam reações adversas a medicamentos no início do tratamento.

DESCRITORES: Fármacos Anti-HIV. Terapia Antirretroviral de Alta Atividade. Qualidade de vida. WHOQOLHIV-bref. Estudos de Coortes. 


\section{INTRODUÇÃO}

No Brasil,nos últimos anos, os esquemas antirretrovirais (ARV) de primeiralinha foram modificados, seguindo a tendência mundial e recomendações da Organização Mundial da Saúde (OMS) ${ }^{1}$. Em 2015, o Protocolo Clínico e Diretrizes Terapêuticas (PCDT) para o manejo do HIV passou a recomendar como esquema de primeira linha um medicamento em dose fixa combinada (DFC) contendo os fármacos tenofovir (TDF), lamivudina (3TC) e efavirenz (EFV).Em 2017, o dolutegravir (DTG), fármaco inibidor da integrase, foi inserido no esquema de primeira linha, em substituição ao EFV, juntamente com um comprimido da associação de TDF e 3TC².

Em países em desenvolvimento, como o Brasil, existem evidências limitadas, em estudos longitudinais, sobre os fatores determinantes da qualidade de vida (QV) em pessoas que vivem com HIV (PVHIV). Em uma revisão de literatura, os fatores sexo, idade, situação familiar, escolaridade, emprego, renda, carga viral, contagem de linfócitos TCD4+, tempo de diagnóstico, presença de sintomas de depressão e ansiedade, apoio social, atenção à saúde, uso de drogas lícitas e ilícitas, adesão à terapia antirretroviral (TARV), estilo de vida e comportamento sexual foram diretamente associados à QV de PVHIV ${ }^{3}$. Portanto, a compreensão da QV é essencial para analisar o impacto físico e biopsicossocial que o HIV pode ocasionar nos indivíduos, possibilitando maior conhecimento acerca de si mesmo, de sua adaptação à condição de viver com HIV e de seu tratamento. Considera-se ainda que existem evidências limitadas sobre o impacto do início da TARV na QV em PVHIV.

O objetivo deste estudo foi avaliar longitudinalmente a QV em PVHIV iniciando a TARV atendidas em três serviços públicos de referência em Belo Horizonte, bem como avaliar os preditores de alteração na QV.

\section{MÉTODOS}

Estudo de coorte, no qual foram utilizados dados do projeto Ecoart, cujos detalhes foram previamente publicados ${ }^{4,5}$.

A seleção da amostra foi não aleatória, e todos os indivíduos elegíveis e presentes em três serviços públicos de assistência especializada em HIV, que dispensam TARV para aproximadamente $80 \%$ das PVHIV no município de Belo Horizonte, foram convidados a participar do estudo. O recrutamento ocorreu entre setembro de 2015 e outubro de 2017.

Foram incluídos indivíduos com até 180 dias de TARV, em acompanhamento em um dos serviços avaliados e que foram identificados por meio do registro no Sistema de Controle Logístico de Medicamentos (Siclom). Os critérios de inclusão foram assinatura do termo de consentimento livre e esclarecido (TCLE), ter 18 anos ou mais, autonomia para responder à entrevista e ter realizado a entrevista basal e a segunda entrevista de acompanhamento.

Os dados foram obtidos em entrevistas face a face e em bases de dados secundárias, utilizando-se formulários padronizados que continham os instrumentos World Health Organization Quality of Life HIV - em sua versão resumida (WHOQOLHIV-bref) -, Escala Hospitalar de Ansiedade e Depressão (HADS) e Escala de Adesão Terapêutica de Morisky de Oito Itens (MMAS-8), todos validados no Brasil ${ }^{6-8}$, além de perguntas relativas a informações sociodemográficas, comportamentais, clínicas, laboratoriais, relacionadas ao tratamento farmacológico e ao serviço de saúde.

Para minimizar potenciais riscos de vieses, os pesquisadores foram devidamente treinados e foi realizado projeto piloto antes de iniciar a coleta de dados.

\section{Variável Dependente}

A QV foi avaliada por meio do instrumento WHOQOLHIV-bref, em dois momentos, na entrevista basal e na segunda entrevista de acompanhamento, prevista para ocorrer seis 
meses após a entrevista basal. A diferença média da QV entre esses dois momentos constituiu a variável dependente deste estudo, que apresenta os resultados unicamente daqueles indivíduos que terminaram o acompanhamento. Em um artigo prévio ${ }^{4}$ foram publicados os dados relativos a QV na entrevista basal.

O WHOQOLHIV-brefé um instrumento específico para avaliação da QV em PVHIV, que, por meio de 31 perguntas, avalia os domínios: físico, psicológico, nível de independência, relações sociais, meio ambiente e espiritualidade. Cada pergunta possui opções de resposta em escala Likert de cinco pontos, onde 1 indica percepções negativas e 5, percepções positivas, exceto para sete itens em que a escala é invertida. A média dos itens dentro de cada domínio é usada para calcular a pontuação total do domínio. O resultado é multiplicado por 4, e, assim, os escores variam entre 4 e $20^{6}$.

O componente QV global e percepção geral da saúde foi construído utilizando as duas primeiras perguntas do instrumento WHOQOLHIV-bref. Fez-se a média dos itens, e o resultado foi multiplicado por 4; dessa forma, os escores também variaram de 4 a $20^{6}$.

\section{Variáveis Independentes}

Para caracterizar a população do estudo, foram analisadas variáveis sociodemográficas, comportamentais, clínicas e laboratoriais, relacionadas ao tratamento farmacológico e ao serviço.

A presença de sinais ou sintomas de ansiedade e depressão foi mensurada por meio da HADS, composta por 14 itens ${ }^{7}$. Pessoas que tiveram 8 ou mais pontos em cada um dos agravos foram classificadas com sintomas de ansiedade e depressão. Os indivíduos foram classificados segundo a presença de sinais e sintomas de ansiedade ou depressão na entrevista basal e na entrevista de acompanhamento, sendo categorizados em: (i) nunca apresentaram sinais e sintomas de ansiedade ou depressão, (ii) apresentaram esses sintomas em alguma das duas entrevistas ou (iii) apresentaram sintomas em ambas as entrevistas.

A MMAS-8 foi utilizada para avaliar a adesão a TARV. O escore total da MMAS-8 varia de $0 \mathrm{a}$ 8 , sendo que, quanto maior for a pontuação, maior é a adesão ${ }^{8}$. Foram considerados aderentes os indivíduos que obtiveram escore de 8 pontos. As reações adversas a medicamentos (RAM) foram avaliadas por meio de autorrelato. Foram considerados indivíduos com carga viral (CV) indetectável aqueles que apresentaram valor inferior a 50 cópias por $\mathrm{ml}$.

A variável classe econômica (status socioeconômico) foi avaliada de acordo com critérios brasileiros, como alta (A, B), intermediária (C) e baixa (D e E), na qual os indivíduos são classificados por meio de grupos socioeconômicos por posse de itens de conforto e nível de escolaridade do chefe familiar?.

Para a variável comorbidade, foi considerado o autorrelato de qualquer doença diagnosticada por médico, como hipertensão arterial, diabetes, hipercolesterolemia, entre outras.

\section{Análise Estatística}

A análise descritiva foi realizada por distribuição de frequências para variáveis categóricas e medidas de tendência central para as variáveis quantitativas.

Para avaliar os escores do instrumento WHOQOLHIV-bref, foram apresentadas médias e desvio-padrão para cada domínio, relativos à entrevista basal e à segunda entrevista de acompanhamento. $\mathrm{O}$ teste $t$ pareado foi utilizado para comparar as diferenças médias dos escores de QV entre as duas entrevistas.

A análise univariável da relação entre variáveis independentes e a alteração no escore de QV foi avaliada pelo teste $t$ Student (comparação de médias) e o teste Mann-Whitney (comparação de medianas). 
A associação foi analisada pelo ajuste de um modelo de regressão linear múltipla. Foram selecionadas para entrar no modelo multivariável as variáveis que apresentaram valor $p$ igual ou inferior a 0,20 .

O método backward stepwise foi utilizado para obtenção do modelo final. Os resultados da regressão linear múltipla foram demonstrados por meio de coeficientes da regressão, com seus respectivos intervalos de confiança a 95\% (IC95\%).

A adequação do modelo foi avaliada por um conjunto de estatísticas. As estatísticas $\mathrm{R}^{2}$ e $\mathrm{R}^{2}$ ajustado foram utilizadas para verificar a porcentagem da variância relacionada ao incremento na QV explicada pelo modelo. A estatística de Durbin- Watson foi utilizada para verificar o pressuposto de que os resíduos não estão correlacionados, com valores entre 0 e 4, sendo que 2 significa ausência de correlação entre os resíduos. Foi testado também se havia multicolinearidade no modelo final, utilizando as estatísticas de tolerância (aceitável $>0,10$ ) e VIF (aceitável < 10) ${ }^{10}$. Para avaliar se os resíduos tinham distribuição normal, foram realizados os seguintes gráficos: resíduos padronizados da regressão por valores previstos da regressão padronizados, histograma de frequências dos resíduos padronizados da regressão, e um gráfico percentil-percentil (P.P. plot).

Foi utilizado o software Statistical Package for the Social Sciences (SPSS) versão 22.0. Em todas as análises considerou-se o nível de significância de 0,05.

\section{Aspectos Éticos}

O projeto de pesquisa Ecoart foi aprovado pelo Comitê de Ética em Pesquisa (COEP) da Universidade Federal de Minas Gerais (protocolo CAAE 31192914.3.3001.5124, parecer CEP 769.085) e dos serviços participantes. A pesquisa foi realizada seguindo instruções da Resolução 466/2012 do Conselho Nacional de Saúde.

\section{RESULTADOS}

Durante todo o período de recrutamento do projeto Ecoart, foram identificados 1.017 indivíduos atendidos nos três serviços. Desses, 468 realizaram a entrevista basal, e $323(69,0 \%)$ realizaram a segunda entrevista de acompanhamento e foram incluídos nesse estudo (Figura 1).

Ao comparar o grupo que completou a segunda entrevista de acompanhamento com aqueles que não a realizaram, não houve diferenças estatisticamente significantes em relação a sexo, idade, estado civil e escolaridade. Também não houve diferenças entre os indivíduos na entrevista basal quanto à QV geral e nos domínios de QV.

A maioria dos entrevistados era do sexo masculino (83,0\%), sem cônjuge (79,9\%), sem filhos (66,9\%), entre 20 e 34 anos (54,5\%), não possuía plano privado de saúde $(72,8 \%)$ e se autodeclarou como não branco (76,9\%). Verificou-se que $80,8 \%$ dos indivíduos residiam com outras pessoas, $38,8 \%$ possuíam de 10 a 12 anos de escolaridade e $59,4 \%$ estavam empregados no momento da entrevista, havendo predomínio da classe econômica C (45,7\%). Em relação às características comportamentais e hábitos de vida, $24,5 \%$ relataram uso atual de tabaco, 65,8\%, uso de álcool recente, e 47,5\% declararam já ter utilizado ou utilizar drogas ilícitas na vida. A maioria declarou ter alguma crença religiosa (79,4\%). Verificou-se que 59,6\% eram homens que fazem sexo com outros homens (HSH). Quanto às características clínicas, 7,8\% apresentaram coinfecção (como tuberculose, sífilis, toxoplasmose e candidíase), 18,3\%, uma ou mais comorbidades autorrelatadas, e 35,9\%, sinais ou sintomas de ansiedade e depressão. A maioria dos indivíduos apresentou classificação clínica assintomática (67,3\%) e tinha tempo de diagnóstico de infecção pelo HIV menor ou igual a seis meses $(61,8 \%)$. No que diz respeito às características laboratoriais basais, $23,5 \%$ apresentaram contagem inicial de linfócitos TCD4+ inferior a 200 células $/ \mathrm{mm}^{3}, 90,1 \%$, CV detectável, e 26,0\%, CV superior a 100.000 cópias $/ \mathrm{ml}$. Em relação ao tratamento farmacológico, 63,2\% utilizaram TDF/3TC/EFV, e 32,2\%, TDF/3TC + DTG. A maioria (52,3\%) estava em uso de TARV há 


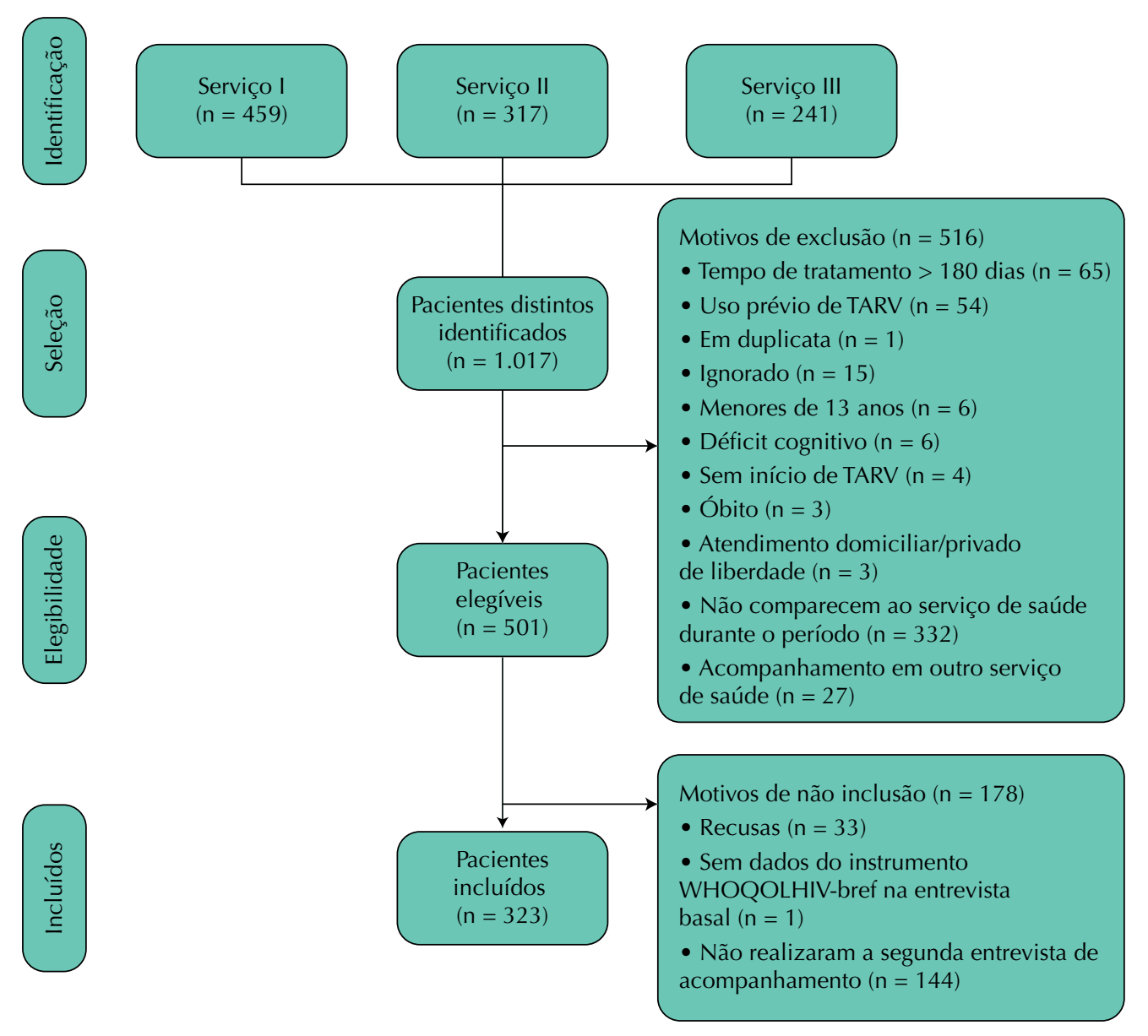

Figura. Diagrama de inclusão dos indivíduos no estudo.

60 dias ou menos, eram não aderentes a TARV $(52,8 \%)$, apresentaram RAM $(85,9 \%)$, com três ou menos RAM $(53,1 \%)$ (Tabela 1$)$.

Na segunda entrevista de acompanhamento, em relação às características clínicas, laboratoriais e relacionadas a TARV, $34,3 \%$ dos indivíduos apresentaram sinais e sintomas de ansiedade e depressão, 73,4\%, CV indetectável (supressão viral), 59,5\% relataram não adesão tratamento, e 51,6\% relataram alguma RAM, sendo que 79,9\% relataram três ou menos RAM (Tabela 1).

Os dados de QV foram obtidos na entrevista basal e na segunda entrevista de acompanhamento, que ocorreu em média 9,47 meses (mediana = 8,66; mínimo = 3,5; máximo = 23,0; intervalo interquartil $=19,5, \mathrm{DP}=3,67$ ) após a entrevista basal.

Foram observadas, ao longo do tempo, melhorias estatisticamente significantes na QV global e nos domínios, físico, psicológico, nível de independência, ambiente e espiritual em indivíduos iniciando TARV. O único domínio em que a diferença média entre as entrevistas basal e de acompanhamento não foi significante foi o das relações sociais.

Os domínios de QV com maiores diferenças médias foram o físico (5,11; DP = -3,75), espiritual $(3,23 ; \mathrm{DP}=6,20)$ e psicológico $(1,32 ; \mathrm{DP}=2,82)($ Tabela 2$)$.

Na análise univariável, indivíduos que relataram uso de drogas ilícitas durante toda a vida apresentaram diferença média na QV mais elevada $(1,25$; DP = 3,19). Indivíduos com ausência de sintomas de ansiedade e depressão apresentaram maior diferença média na QV $(2,78 ; \mathrm{DP}=3,22)$. Dentre as variáveis laboratoriais e relacionadas a TARV, não foi observada associação estatisticamente significativa entre CV, esquema ARV (TDF/3TC/ 
Tabela 1. Características sociodemográficas, comportamentais, clínicas, laboratoriais, terapêuticas e relacionadas ao serviço de PVHIV em TARV inicial na entrevista basal e na segunda entrevista de acompanhamento, Belo Horizonte, Minas Gerais $(\mathrm{n}=323)$.

\begin{tabular}{|c|c|c|}
\hline \multicolumn{3}{|l|}{ Entrevista basal } \\
\hline Características & $\mathbf{n}$ & $(\%)$ \\
\hline \multicolumn{3}{|l|}{ Sociodemográficas } \\
\hline Sexo (masculino) & 268 & 83 \\
\hline \multicolumn{3}{|l|}{ Idade (anos) } \\
\hline $18-19$ & 10 & 3,1 \\
\hline $20-34$ & 176 & 54,5 \\
\hline $35-49$ & 102 & 31,6 \\
\hline$\geq 50$ & 35 & 10,8 \\
\hline Idade (média (DP)), anos & \multicolumn{2}{|c|}{$34,5(10,7)$} \\
\hline Estado civil (solteiro/divorciado/viúvo) & 258 & 79,9 \\
\hline Cor de pele (não branco) & 246 & 76,9 \\
\hline \multicolumn{3}{|l|}{ Escolaridade (anos) } \\
\hline$\leq 9$ & 75 & 23,3 \\
\hline $10-12$ & 125 & 38,8 \\
\hline$\geq 13$ & 122 & 37,9 \\
\hline Filhos (não) & 216 & 66,9 \\
\hline Reside com outras pessoas (sim) & 261 & 80,8 \\
\hline Emprego (sim) & 192 & 59,4 \\
\hline Plano de saúde (não) & 235 & 72,8 \\
\hline \multicolumn{3}{|l|}{ Classe econômica } \\
\hline A, B & 122 & 38,7 \\
\hline $\mathrm{C}$ & 144 & 45,7 \\
\hline $\mathrm{D}, \mathrm{E}$ & 49 & 15,6 \\
\hline \multicolumn{3}{|l|}{ Comportamentais } \\
\hline Crença religiosa (sim) & 255 & 79,4 \\
\hline Tabagismo atual (sim) & 79 & 24,5 \\
\hline Consumo de álcool no último mês (sim) & 212 & 65,8 \\
\hline Uso de droga ilícita na vida (sim) & 153 & 47,5 \\
\hline Categoria de risco/exposição (HSH) & 168 & 59,6 \\
\hline \multicolumn{3}{|l|}{ Clínicas } \\
\hline Classificação clínica (assintomática) & 214 & 67,3 \\
\hline Sinais ou sintomas de ansiedade ou depressão (sim) & 116 & 35,9 \\
\hline Comorbidades autorrelatadas (não) & 264 & 81,7 \\
\hline Coinfecções autorrelatadas (não) & 296 & 92,2 \\
\hline Tempo de diagnóstico de HIV (média (DP)), meses & \multicolumn{2}{|c|}{$77,1(59,8)$} \\
\hline \multicolumn{3}{|l|}{ Laboratoriais } \\
\hline \multicolumn{3}{|l|}{ Contagem de linfócitos TCD4+ (células/mm3) } \\
\hline$<200$ & 76 & 23,5 \\
\hline $200-500$ & 124 & 38,4 \\
\hline$>500$ & 96 & 29,7 \\
\hline Dados faltantes & 27 & 8,4 \\
\hline \multicolumn{3}{|l|}{$\mathrm{CV}^{*}$} \\
\hline Detectável & 291 & 90,1 \\
\hline Indetectável & 5 & 1,5 \\
\hline Dados faltantes & 27 & 8,4 \\
\hline \multicolumn{3}{|l|}{ CV (cópias/mL) } \\
\hline$\leq 100.000$ & 212 & 65,6 \\
\hline
\end{tabular}


Tabela 1. Características sociodemográficas, comportamentais, clínicas, laboratoriais, terapêuticas e relacionadas ao serviço de PVHIV em TARV inicial na entrevista basal e na segunda entrevista de acompanhamento, Belo Horizonte, Minas Gerais ( $\mathrm{n}=323)$. (Continuação)

\begin{tabular}{|c|c|c|}
\hline \multicolumn{3}{|l|}{ Entrevista basal } \\
\hline Características & $\mathbf{n}$ & $(\%)$ \\
\hline$>100.000$ & 84 & 26 \\
\hline Dados faltantes & 27 & 8,4 \\
\hline \multicolumn{3}{|l|}{ Relacionadas à TARV } \\
\hline \multicolumn{3}{|l|}{ Esquema antirretroviral } \\
\hline TDF/3TC/EFV & 204 & 63,2 \\
\hline TDF/3TC + DTG & 104 & 32,2 \\
\hline Outros esquemas & 15 & 4,6 \\
\hline Tempo de tratamento ART (média (DP)), dias & \multicolumn{2}{|c|}{$77,1(59,8)$} \\
\hline Adesão (não) & 163 & 52,8 \\
\hline RAM (sim) & 267 & 85,9 \\
\hline Número de RAM (média (DP)) & \multicolumn{2}{|c|}{$3,8(3,2)$} \\
\hline \multicolumn{3}{|l|}{ Relacionadas ao serviço } \\
\hline \multicolumn{3}{|l|}{ Serviço de saúde de acompanhamento } \\
\hline I & 106 & 32,8 \\
\hline II & 144 & 44,6 \\
\hline III & 73 & 22,6 \\
\hline \multicolumn{3}{|l|}{ Segunda entrevista de acompanhamento } \\
\hline \multicolumn{3}{|l|}{ Clínicas } \\
\hline \multicolumn{3}{|l|}{ Sinais ou sintomas de ansiedade ou depressão } \\
\hline $\begin{array}{l}\text { Nunca (nem na basal, nem na entrevista de } \\
\text { acompanhamento) }\end{array}$ & 41 & 12,7 \\
\hline Alguma vez (basal ou entrevista de acompanhamento) & 244 & 75,5 \\
\hline Sempre (basal e entrevista de acompanhamento) & 36 & 11.1 \\
\hline \multicolumn{3}{|l|}{ Laboratoriais } \\
\hline \multicolumn{3}{|l|}{ CV (supressão viral) } \\
\hline Detectável & 43 & 13,3 \\
\hline Indetectável & 237 & 73,4 \\
\hline Dados faltantes & 43 & 13,3 \\
\hline \multicolumn{3}{|l|}{ Relacionadas à TARV } \\
\hline Adesão (não) & 191 & 59,5 \\
\hline RAM (sim) & 165 & 51,6 \\
\hline Número de RAM (média (DP)) & \multicolumn{2}{|c|}{$1,9(2,7)$} \\
\hline
\end{tabular}

LT-CD4+: Linfócitos T-CD4+; HSH: homens que fazem sexo com homens; TDF: tenofovir; 3TC: lamivudina; EFV: efavirenz; DTG: dolutegravir; TARV: terapia antirretroviral; RAM: reação adversa a medicamento; HIV: human immunodeficiency virus; Outros riscos: hemofílicos, transfusão e ocupacional.

* CV indetectável: < 50 cópias/ml.

EFV; TDF/3TC + DTG e outros esquemas), tempo de tratamento, adesão ao tratamento, RAM, número de RAM e alteração da QV média (Tabela 3).

No modelo final de regressão linear múltipla, apresentaram aumento na QV indivíduos que possuíam alguma crença religiosa $(0,92$; IC95\% 0,20 a 1,64) e os que residiam com outras pessoas $(1,00$; IC95\% 0,26 a 1,65). Enquanto indivíduos que reportaram sinais ou sintomas de ansiedade e depressão alguma vez (-0,99; IC95\% -1,89 a -0,10) ou sempre (-2,91; IC95\% -4,11 a -1,70) apresentaram redução na QV. A cada RAM adicional relatada na entrevista basal houve redução na QV do indivíduo (-0,09; IC95\% -0,18 a 0,01). Aqueles indivíduos que apresentaram maiores escores de QV na entrevista basal obtiveram menores incrementos de QV ao final do estudo, mostrando assim uma associação inversamente proporcional $(-0,60 ;$ IC95\% -0,69 a -0,50) (Tabela 4). 
Tabela 2. Distribuição dos escores dos domínios do WHOQOLHIV-bref na entrevista basal e após o acompanhamento em indivíduos vivendo com HIV em TARV inicial, Belo Horizonte, Minas Gerais $(\mathrm{n}=323)$.

\begin{tabular}{|c|c|c|c|c|c|}
\hline $\begin{array}{l}\text { Domínios } \\
\text { WHOQOLHIV-brefa }\end{array}$ & $\mathbf{n}$ & $\begin{array}{l}\text { Entrevista basal } \\
\text { - média (DP) }\end{array}$ & $\begin{array}{c}\text { Segunda entrevista } \\
\text { de acompanhamento } \\
\text { - média (DP) }\end{array}$ & $\begin{array}{c}\text { Diferença da média } \\
\text { da segunda entrevista } \\
\text { e da entrevista basal } \\
\text { (DP) }\end{array}$ & $\mathbf{p}$ \\
\hline $\begin{array}{l}\text { Qualidade de vida } \\
\text { global }\end{array}$ & 323 & $15,37(3,12)$ & $16,24(2,93)$ & $0,86(3,28)$ & $<0,001^{\mathrm{b}}$ \\
\hline Físico & 314 & $10,94(2,20)$ & $16,04(2,94)$ & $5,11(3,75)$ & $<0,001^{\mathrm{b}}$ \\
\hline Psicológico & 318 & $14,03(2,06)$ & $15,35(2,73)$ & $1,32(2,82)$ & $<0,001^{\mathrm{b}}$ \\
\hline $\begin{array}{l}\text { Nível de } \\
\text { independência }\end{array}$ & 316 & $14,89(2,26)$ & $15,72(2,86)$ & $0,82(3,08)$ & $<0,001^{b}$ \\
\hline Relações sociais & 295 & $15,28(3,01)$ & $15,57(2,83)$ & $0,29(3,15)$ & 0,113 \\
\hline Ambiente & 315 & $14,29(2,40)$ & $14,71(2,36)$ & $0,42(2,08)$ & $<0,001^{b}$ \\
\hline Espiritual & 315 & $11,67(3,42)$ & $14,89(3,73)$ & $3,23(6,20)$ & $<0,001^{\mathrm{b}}$ \\
\hline
\end{tabular}

DP: desvio-padrão; TARV: terapia antirretroviral.

a Escores dos domínios variam de 4 a 20 (maiores escores correspondem a melhor qualidade de vida).

${ }^{\mathrm{b}}$ Estatisticamente significante.

Tabela 3. Análise univariável de fatores associados com a diferença média da qualidade de vida (QV) geral em PVHIV em TARV inicial, Belo Horizonte, Minas Gerais $(\mathrm{n}=323)$.

\begin{tabular}{|c|c|c|c|c|c|}
\hline & n & $\begin{array}{l}\text { Entrevista basal } \\
\text { - média (DP) }\end{array}$ & $\begin{array}{c}\text { Segunda } \\
\text { entrevista de } \\
\text { acompanhamento } \\
\text { - média (DP) }\end{array}$ & $\begin{array}{l}\text { Diferença na } \\
\text { QV - média } \\
\text { (DP) }\end{array}$ & $\mathbf{p}^{b}$ \\
\hline \multicolumn{6}{|l|}{ Sociodemográficas } \\
\hline \multicolumn{6}{|l|}{ Sexo } \\
\hline Masculino & 268 & $15,69(2,84)$ & $16,39(2,82)$ & $0,70(3,23)$ & \multirow{2}{*}{0,05} \\
\hline Feminino & 55 & $13,85(3,92)$ & $15,49(3,34)$ & $1,64(3,47)$ & \\
\hline Idade (ano adicional) & 323 & $-0,01(0,02)$ & $-0,02(0,02)$ & $-0,01(0,02)$ & 0,629 \\
\hline \multicolumn{6}{|l|}{ Estado civil } \\
\hline Solteiro, divorciado, viúvo & 258 & $15,23(3,16)$ & $16,21(2,81)$ & $0,98(3,30)$ & \multirow{2}{*}{0,177} \\
\hline Casado, união estável & 65 & $15,94(2,89)$ & $16,31(3,36)$ & $0,37(3,18)$ & \\
\hline \multicolumn{6}{|l|}{ Cor de pele } \\
\hline Branco & 74 & $16,14(2,57)$ & $16,89(2,75)$ & $0,76(3,05)$ & \multirow{2}{*}{0,807} \\
\hline Não branco & 246 & $15,17(3,19)$ & $16,03(2,97)$ & $0,86(3,30)$ & \\
\hline \multicolumn{6}{|l|}{ Escolaridade (anos) } \\
\hline$\leq 9$ & 75 & $14,29(3,36)$ & $15,15(3,71)$ & $0,85(4,04)$ & \multirow{2}{*}{0,932} \\
\hline de 10 a 12 & 125 & $15,34(3,01)$ & $16,24(2,60)$ & $0,90(2,98)$ & \\
\hline$\geq 12$ & 122 & $16,07(2,91)$ & $16,87(2,49)$ & $0,80(3,08)$ & 0,922 \\
\hline \multicolumn{6}{|l|}{ Filhos } \\
\hline Não & 216 & $15,66(2,99)$ & $16,50(2,73)$ & $0,84(3,18)$ & \multirow{2}{*}{0,05} \\
\hline Sim & 107 & $14,80(3,31)$ & $15,70(3,24)$ & $0,90(3,49)$ & \\
\hline \multicolumn{6}{|l|}{ Reside com outras pessoas } \\
\hline Não & 62 & $15,26(3,01)$ & $15,39(3,02)$ & $0,13(3,76)$ & \multirow{2}{*}{0,05} \\
\hline Sim & 261 & $15,40(3,15)$ & $16,44(2,87)$ & $1,03(3,14)$ & \\
\hline \multicolumn{6}{|l|}{ Emprego } \\
\hline Não & 131 & $14,58(3,41)$ & $15,80(3,19)$ & $1,22(3,36)$ & \multirow[t]{2}{*}{$0,10^{3}$} \\
\hline Sim & 192 & $15,92(2,78)$ & $16,53(2,70)$ & $0,61(3,21)$ & \\
\hline \multicolumn{6}{|l|}{ Plano de saúde } \\
\hline Não & 235 & $15,23(3,08)$ & $16,08(2,51)$ & $0,84(3,43)$ & \multirow{2}{*}{0,871} \\
\hline Sim & 88 & $15,75(3,22)$ & $16,66(2,51)$ & $0,91(2,86)$ & \\
\hline \multicolumn{6}{|l|}{ Classe econômica } \\
\hline$C, D, E$ & 193 & $15,10(3,19)$ & $15,95(3,06)$ & $0,85(3,43)$ & \multirow{2}{*}{0,99} \\
\hline$A, B$ & 122 & $15,92(3,94)$ & $16,77(2,53)$ & $0,85(3,03)$ & \\
\hline
\end{tabular}


Tabela 3. Análise univariável de fatores associados com a diferença média da qualidade de vida (QV) geral em PVHIV em TARV inicial, Belo Horizonte, Minas Gerais ( $\mathrm{n}=323)$. (Continuação)

\begin{tabular}{|c|c|c|c|c|c|}
\hline & $\mathbf{n}$ & $\begin{array}{l}\text { Entrevista basal } \\
\text { - média (DP) }\end{array}$ & $\begin{array}{c}\text { Segunda } \\
\text { entrevista de } \\
\text { acompanhamento } \\
\text { - média (DP) }\end{array}$ & $\begin{array}{l}\text { Diferença na } \\
\text { QV - média } \\
\text { (DP) }\end{array}$ & $\mathbf{p}^{\mathbf{b}}$ \\
\hline \multicolumn{6}{|l|}{ Comportamentais } \\
\hline \multicolumn{6}{|l|}{ Crença religiosa } \\
\hline Não & 66 & $15,52(3,42)$ & $15,79(3,24)$ & $0,27(3,61)$ & \multirow{2}{*}{0,096} \\
\hline Sim & 255 & $15,31(3,04)$ & $16,34(2,84)$ & $1,03(3,18)$ & \\
\hline \multicolumn{6}{|c|}{ Tabagismo atual (entrevista basal) } \\
\hline Não & 244 & $15,79(2,78)$ & $16,48(2,65)$ & $0,69(3,04)$ & \multirow{2}{*}{0,098} \\
\hline Sim & 79 & $14,10(3,73)$ & $15,49(3,56)$ & $1,39(3,91)$ & \\
\hline \multicolumn{6}{|c|}{ Consumo de álcool no mês anterior à entrevista basal } \\
\hline Não & 110 & $15,47(3,03)$ & $16,16(3,01)$ & $0,69(3,34)$ & \multirow{2}{*}{0,514} \\
\hline Sim & 212 & $15,33(3,18)$ & $16,27(2,90)$ & $0,94(3,26)$ & \\
\hline \multicolumn{6}{|c|}{ Uso de droga ilícita na vida } \\
\hline Não & 169 & $15,66(2,95)$ & $16,17(2,95)$ & $0,51(3,34)$ & \multirow{2}{*}{$0,042^{\mathrm{a}}$} \\
\hline Sim & 153 & $15,11(3,24)$ & $16,37(2,84)$ & $1,25(3,19)$ & \\
\hline \multicolumn{6}{|c|}{ Categoria de risco/exposição } \\
\hline Outros & 114 & $14,84(3,42)$ & $15,72(2,93)$ & $0,88(3,31)$ & \multirow[t]{2}{*}{0,984} \\
\hline $\mathrm{HSH}$ & 168 & $15,73(2,89)$ & $16,60(2,69)$ & $0,87(3,27)$ & \\
\hline \multicolumn{6}{|l|}{ Clínicas } \\
\hline \multicolumn{6}{|c|}{ Classificação clínica } \\
\hline Aids & 58 & $15,66(3,00)$ & $16,28(3,38)$ & $0,62(3,84)$ & \multirow[b]{2}{*}{0,539} \\
\hline $\begin{array}{l}\text { Sintomático ou } \\
\text { assintomático }\end{array}$ & 260 & $15,29(3,11)$ & $16,21(2,82)$ & $0,92(3,17)$ & \\
\hline
\end{tabular}

Presença de sinais ou sintomas de ansiedade ou depressão durante o acompanhamento

$\begin{array}{lccccc}\text { Nunca } & 41 & 13,71(3,27) & 16,49(2,27) & 2,78(3,22) & \\ \text { Alguma vez } & 244 & 15,55(3,12) & 16,39(2,88) & 0,84(3,05) & <0,001^{\text {a }} \\ \text { Sempre } & 36 & 16,00(2,29) & 14,78(3,55) & -1,22(3,71) & <0,001^{\text {a }}\end{array}$

Comorbidades autorrelatadas

$\begin{array}{lccccc}\text { Não } & 264 & 15,62(2,99) & 16,45(2,61) & 0,83(3,12) & 0,752 \\ \text { Sim } & 59 & 14,27(3,47) & 15,25(3,93) & 0,98(3,97) & \end{array}$

Coinfecções autorrelatadas

Não $\quad 296 \quad 15,46(3,16) \quad 16,35(2,84) \quad 0,89(3,21)$

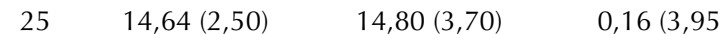

Tempo de diagnóstico do $322<0,01(0,01) \quad<0,01(0,01) \quad<-0,01(0,01) \quad 0,675$

HIV (mês adicional)

Laboratoriais

CV - entrevista basal

Detectável $291 \quad 15,43(3,15) \quad 16,26(2,93) \quad 0,83(3,30)$

Indetectável $\quad 5 \quad 12,80(2,28) \quad 16,40(2,19) \quad 3,60(3,29)$

$\mathrm{CV}$ - segunda entrevista de acompanhamento ${ }^{c}$

$\begin{array}{lccccc}\begin{array}{l}\text { Detectável } \\ \text { Indetectável }\end{array} & 43 & 14,65(3,14) & 16,14(2,37) & 1,49(3,03) & 0,085 \\ \text { CV (cópias/mL) } & 237 & 15,66(2,93) & 16,20(3,00) & 0,54(3,35) & \\ \text { Até } 100 \mathrm{mil} & & & & & \\ \text { Acima de } 100 \mathrm{mil} & 212 & 15,31(3,18) & 16,22(2,88) & 0,91(3,30) & 0,823\end{array}$

Relacionadas à TARV

Esquema antirretroviral

\begin{tabular}{llllll} 
TDF/3TC/EFV & 204 & $15,27(3,18)$ & $16,11(2,82)$ & $0,83(3,44)$ & 0,635 \\
TDF/3TC + DTG & 104 & $15,75(2,96)$ & $16,77(2,59)$ & $1,02(2,83)$ & \\
\hline
\end{tabular}


Tabela 3. Análise univariável de fatores associados com a diferença média da qualidade de vida (QV) geral em PVHIV em TARV inicial, Belo Horizonte, Minas Gerais ( $\mathrm{n}=323)$. (Continuação)

\begin{tabular}{|c|c|c|c|c|c|}
\hline & $\mathbf{n}$ & $\begin{array}{l}\text { Entrevista basal } \\
\text { - média (DP) }\end{array}$ & $\begin{array}{c}\text { Segunda } \\
\text { entrevista de } \\
\text { acompanhamento } \\
\text { - média (DP) }\end{array}$ & $\begin{array}{l}\text { Diferença na } \\
\text { QV - média } \\
\text { (DP) }\end{array}$ & $\mathbf{p}^{\mathbf{b}}$ \\
\hline Outros esquemas & 15 & $14,13(3,16)$ & $14,27(5,06)$ & $0,13(4,10)$ & 0,453 \\
\hline $\begin{array}{l}\text { Tempo de tratamento ART } \\
\text { (dia adicional) }\end{array}$ & 323 & $<-0,01(<0,01)$ & $<-0,01(<0,01)$ & $<-0,01(<0,01)$ & 0,306 \\
\hline \multicolumn{6}{|l|}{ Adesão - entrevista basal } \\
\hline Não & 163 & $15,10(3,27)$ & $15,73(3,24)$ & $0,63(3,43)$ & \multirow{2}{*}{0,270} \\
\hline Sim & 146 & $15,71(2,98)$ & $16,75(2,50)$ & $1,04(3,14)$ & \\
\hline \multicolumn{6}{|c|}{ Adesão - segunda entrevista de acompanhamento } \\
\hline Não & 191 & $15,20(3,38)$ & $15,92(3,04)$ & $0,71(3,44)$ & \multirow{2}{*}{0,39} \\
\hline Sim & 130 & $15,68(2,67)$ & $16,71(2,73)$ & $1,03(3,04)$ & \\
\hline \multicolumn{6}{|l|}{ RAM - entrevista basal } \\
\hline Não & 44 & $16,46(3,22)$ & $17,00(2,42)$ & $0,55(3,09)$ & \multirow{2}{*}{0,503} \\
\hline Sim & 267 & $15,18(3,12)$ & $16,09(3,02)$ & $0,91(3,35)$ & \\
\hline \multicolumn{6}{|c|}{ RAM - segunda entrevista de acompanhamento } \\
\hline Não & 155 & $15,79(2,63)$ & $16,66(2,76)$ & $0,86(3,13)$ & \multirow{2}{*}{0,913} \\
\hline Sim & 165 & $15,04(3,48)$ & $15,87(3,05)$ & $0,82(3,44)$ & \\
\hline $\begin{array}{l}\text { RAM adicional - Entrevista } \\
\text { basal }\end{array}$ & 311 & $-0,26(0,06)$ & $-0,16(0,05)$ & $0,10(0,97)$ & 0,086 \\
\hline $\begin{array}{l}\text { RAM adicional - } \\
\text { Segunda entrevista de } \\
\text { acompanhamento }\end{array}$ & 323 & $-0,23(0,06)$ & $-0,27(0,06)$ & $-0,04(0,07)$ & 0,527 \\
\hline
\end{tabular}

QV: qualidade de vida; PVHIV: pessoa vivendo com HIV; DP = desvio-padrão; LT-CD4+: linfócitos T-CD4+; HSH: homens que fazem sexo com homens; TDF: tenofovir; 3TC: lamivudina; EFV: efavirenz; DTG: dolutegravir; TARV: terapia antirretroviral; RAM: reação adversa a medicamento; HIV: human immunodeficiency virus; Outros riscos: hemofílicos, transfusão e ocupacional.

a Estatisticamente significante.

${ }^{\text {b }}$ Os valores de $p$ se referem à comparação entre as diferenças no incremento de QV de acordo com cada variável.

${ }^{c}$ CV indetectável: < 50 cópias $/ \mathrm{ml}$.

Tabela 4. Modelo multivariável final dos fatores associados com a diferença na QV geral em PVHIV em TARV inicial, Belo Horizonte, Minas Gerais $\left(n=307^{*}\right)$.

\begin{tabular}{lcccc}
\hline & Coeficiente & IC95\% & p \\
\hline Constante & 9,887 & 8,006 & 11,768 & $<0,001$ \\
Crença (sim) & 0,922 & 0,199 & 1,645 & 0,013 \\
Reside com outras pessoas (sim) & 1,00 & 0,26 & 1,65 & 0,008 \\
$\begin{array}{l}\text { Sinais ou sintomas de ansiedade ou } \\
\text { depressão (alguma vez versus nunca) }\end{array}$ & $-0,993$ & $-1,891$ & $-0,096$ & 0,0030 \\
$\begin{array}{l}\text { Sinais ou sintomas de ansiedade ou } \\
\text { depressão (sempre versus nunca) }\end{array}$ & $-2,906$ & $-4,111$ & $-1,701$ & 0,000 \\
$\begin{array}{l}\text { Reação adversa adicional reportada } \\
\text { na entrevista basal }\end{array}$ & $-0,088$ & $-0,184$ & 0,007 & 0,070 \\
$\begin{array}{l}\text { Escore adicional na QV Global } \\
\text { reportada na entrevista basal }\end{array}$ & $-0,596$ & $-0,692$ & $-0,500$ & $<0,001$ \\
\hline
\end{tabular}

QV: qualidade de vida; PVHIV: pessoa vivendo com HIV; TARV: terapia antirretroviral.

* 16 pacientes com dados faltantes em covariáveis foram excluídos do modelo final.

Com relação à adequação do modelo de regressão linear múltipla, a estatística de $\mathrm{R}^{2}$ ajustado foi igual a 0,414 , ou seja, o modelo explicou aproximadamente $41 \%$ da variância do incremento da QV. A estatística de Durbin-Watson foi igual a 2,02, indicando que não houve correlação entre os resíduos. Nas estatísticas de colinearidade, todos os preditores tiveram valores de tolerância acima de 0,56 e valores de VIF próximos de $1(1,00$ a 1,78). Foi verificada também a distribuição dos resíduos padronizados, sendo que se aproximaram da distribuição normal. Essa suposição foi também confirmada pelo gráfico P-P normal de regressão dos resíduos. Finalmente, o gráfico de dispersão entre os resíduos padronizados 
e previstos não mostrou que eles estavam aleatoriamente distribuídos; portanto, o modelo desenvolvido apresenta bom ajuste.

\section{DISCUSSÃO}

Os indivíduos em início de TARV desta coorte apresentaram incremento na QV, sendo que possuir crença e morar com outras pessoas associaram-se positivamente à QV, enquanto possuir sinais ou sintomas de ansiedade e depressão, alguma vez ou sempre, e RAM adicional reportada foram preditores associados a pior QV.

As características dessa população foram semelhantes às de outros estudos ${ }^{11,12}$ e boletins epidemiológicos ${ }^{13,14}$, com predominância de indivíduos do sexo masculino, jovens, cor de pele autodeclarada não branca e com alta escolaridade.

O presente estudo mostrou que, ao longo do tempo, indivíduos em uso inicial de TARV apresentaram aumento no escore global de $\mathrm{QV}$, consonante com pesquisas anteriores ${ }^{15-17}$.

A maior diferença média de QV observada no domínio físico, no qual são avaliados aspectos como dor, desconforto, energia, sono e repouso, pode estar relacionada ao uso de TARV. Esta provoca alterações no curso da infecção, como desaceleração da progressão da imunodeficiência provocada pelo vírus HIV e restauração da resposta imunológica dos indivíduos - consequentemente, pode impactar positivamente na vida das pessoas ${ }^{18}$.

No domínio relações sociais, em que foram avaliadas questões relacionadas a aceitação do diagnóstico, apoio familiar, relacionamento interpessoal e vida sexual, foi observada menor diferença média de QV. A relação social é um complicador para as PVHIV, uma vez que a infecção pelo vírus pode ser uma condição de saúde estigmatizante, que envolve sentimentos de discriminação e, consequentemente, falta de apoio social e sentimentos de solidão. Considerando que esses fatores dificilmente serão influenciados pelo uso da TARV, é importante ressaltar a necessidade de melhorar as relações pessoais e o suporte social para esses indivíduos ${ }^{15}$.

Foi demonstrado aumento estatisticamente significante no componente de percepção global da QV. Os benefícios dos medicamentos na QV podem ser explicados pela redução dos sintomas clínicos da infecção. Ou seja, isso reforça a necessidade de vinculação e retenção dos indivíduos ao serviço de saúde e adesão ao tratamento.

Alguns fatores comportamentais, sociodemográficos, clínicos e relacionados a TARV mostraram relação com a alteração na QV em indivíduos no início da TARV, sendo esses achados condizentes com a literatura científica ${ }^{15-17,19,20}$.

Sentimentos de culpa, solidão e receio da morte, comumente reportados por PVHIV, estão relacionados ao exercício da espiritualidade e crenças religiosas ${ }^{12,21}$, o que contribui para o aumento da QV e da saúde do indivíduo. Fé e crenças religiosas são importantes estratégias de enfrentamento de condições de saúde como o HIV, quando aliadas a TARV ${ }^{11,21}$.

Nossos resultados destacam a importância do envolvimento familiar para a redução do estigma e do preconceito relacionados ao HIV. Em outro estudo utilizando o mesmo instrumento, porém com delineamento transversal ${ }^{22}$, realizado na região Sul do Brasil, foi demonstrado que os indivíduos que moravam sozinhos apresentaram $30 \%$ de propensão a pior QV no domínio relações sociais. Um menor suporte emocional durante o tratamento poderia explicar parcialmente os resultados encontrados. Outro estudo transversal, realizado com 100 PVHIV no Nepal, utilizando o instrumento WHOQOL-bref, demonstrou que um bom apoio familiar tem maior impacto no incremento de QV, sendo estatisticamente associado à QV geral ${ }^{23}$.

Sinais ou sintomas de ansiedade e depressão são fatores que podem limitar a QV de PVHIV ${ }^{24}$. Existem evidências de que depressão e ansiedade são mais frequentes em PVHIV do que 
na população em geral ${ }^{25}$, o que pode exercer influência negativa no comportamento do indivíduo e contribuir para dificuldades em relação ao tratamento e ainda para pior adesão a TARV $^{26}$. O impacto de sintomas psiquiátricos na QV de PVHIV também acontece devido a uma deterioração do sistema imunológico e consequente aumento da progressão da doença, justificados pelo nível aumentado de estresse e sintomas depressivos ${ }^{27}$. Apesar de a HADS não ser utilizada com fins diagnósticos, e sim para avaliar sinais e sintomas de ansiedade e depressão, foi observada associação entre esses sinais e sintomas e a redução da QV nas PVHIV nas entrevistas de acompanhamento, o que evidencia a importância do acompanhamento por um profissional de saúde (psicólogo, psiquiatra, entre outros) nos serviços onde esses indivíduos estão sendo acompanhados. Em outro estudo sobre QV do grupo Ecoart, de delineamento transversal, realizado com 366 indivíduos em Belo Horizonte, estiveram associados a menor QV indivíduos solteiros, com outras comorbidades, com menor nível educacional, tabagistas, com sinais e sintomas de ansiedade e depressão ${ }^{4}$.Em outro estudo, com delineamento transversal com PVHIV, realizado em Camboja, onde foram avaliadas 150 pessoas, os autores sugerem forte associação entre o incremento da QV avaliado pelo escore global de QV e pelos seis domínios do WHOQOLHIV-brefe a ausência de sinais ou sintomas de ansiedade e depressão ${ }^{28}$.

Neste estudo, observou-se que a cada RAM adicional relatada por indivíduos em tratamento houve maior redução na QV, ou seja, quanto maior é o número de RAM relatadas, maior é a redução na QV. Este resultado é similar ao de outro estudo realizado no Brasil, que descreveu o menor número de RAM a TARV como um dos fatores associados à melhora da $\mathrm{QV}^{29}$. A ocorrência de RAM pode afetar física e psicologicamente a realização de atividades profissionais e pessoais e, consequentemente, a QV de indivíduos em tratamento ${ }^{5}$. A monitorização do uso de antirretrovirais é importante ferramenta para avaliar a segurança desses medicamentos na prática clínica ${ }^{5}$ e garantir melhor assistência e satisfação dos indivíduos com o tratamento ${ }^{29}$.

Ao compararmos os indivíduos em uso do esquema TDF/3TC + DTG com aqueles em uso de TDF/3TC/EFV ou outros esquemas, não foram observadas diferenças estatisticamente significantes na diferença média de QV geral, o que pode ser explicado por parte da população do estudo Ecoart ter iniciado o uso dos ARV mesmo assintomático, em função da mudança do protocolo de tratamento de HIV pelo MS. Portanto, esses indivíduos podem não ter reconhecido a melhora na QV com o uso da TARV, independentemente do esquema utilizado. Este estudo apresenta como limitação a incompletude dos dados do Sistema de Controle de Exames Laboratoriais (Siscel), como contagem de linfócitos TCD4+ e CV. Para minimizar essa limitação, consideramos os dados do período de três meses anteriores e posteriores à segunda entrevista de acompanhamento, resultando em baixo percentual de dados faltantes.

Como pontos positivos, deve-se ressaltar que o estudo élongitudinal, que a coleta de dados foi realizada com rigor metodológico e em locais representativos da PVHIV de Belo Horizonte, o número elevado da amostra e a robustez do modelo final obtido por meio de análise multivariável. Além disso, o WHOQOLHIV-brefé um instrumento que avalia a QV nas duas semanas anteriores à entrevista; assim, potenciais vieses de memória foram minimizados.

Novos estudos em PVHIV com maior tempo de seguimento, utilizando o WHOQOLHIV-bref, bem como outros instrumentos, são necessários para mensurar as alterações na QV desses indivíduos e contribuir para o direcionamento de ações e intervenções dos profissionais de saúde que podem contribuir para aumentar a QV em PVHIV.

Conclui-se que, ao longo do tempo, PVHIV iniciando a TARV apresentaram melhora na QV. Há necessidade de acompanhar e prover cuidados de saúde, em especial para indivíduos com sinais e sintomas de ansiedade e depressão e que relatam reações adversas no início do tratamento. 
Este estudo pode contribuir para o planejamento e o direcionamento de ações e políticas públicas, bem como para identificar fatores modificáveis que possam aumentar a QV desses indivíduos.

\section{REFERÊNCIAS}

1. World Health Organization. Consolidated guidelines on the use of antiretroviral drugs for treating and preventing HIV infection: recommendations for a public health approach. 2. ed. Genebra: WHO; 2016 [citado 20 maio 2018]. Disponível em: http://www.who.int/hiv/pub/arv/ arv-2016/en/.

2. Brasil. Ministério da Saúde, Secretaria de Vigilância em Saúde, Departamento de Vigilância, Prevenção e Controle das Infecções Sexualmente Transmissíveis, do HIV/aids e das Hepatites Virais. Protocolo clínico e diretrizes terapêuticas para manejo da infecção pelo HIV em adultos. Brasília; 2017.

3. Degroote S, Vogelaers D, Vandijck DM. What determines health-related quality of life among people living with HIV: an updated review of the literature. Arch Public Health. 2014;72(1). https://doi.org/10.1186/2049-3258-72-40.

4. Costa JO, Pearson SA, Acurcio FA, Bonolo PF, Silveira MR, Ceccato MGB. Health-related quality of life among HIV-infected patients initiating treatment in Brazil in the single-tablet regimen era. AIDS Care. 2019;31(5):572-81. https://doi.org/10.1080/09540121.2019.1576841.

5. Mendes JC et al. Adverse reactions associated with first-line regimens in patient initiating antiretroviral therapy. European Journal of Clinical Pharmacology. 2018;74(8):1077-88. https:// doi.org/10.1007/s00228-018-2472.

6. Zimpel RR, Fleck MP. (2007). Quality of life in HIV-positive Brazilians: application and validation of the WHOQOL-HIV, Brazilian version. AIDS Care. 2007;19(7), 923-30. https://doi. org/10.1080/09540120701213765.

7. Botega NJ, Bio MR, Zomignani MA, Garcia Jr. C, Pereira WAB. Transtornos do humor em enfermaria de clínica médica e validação de escala de medida (HADS) de ansiedade e depressão. Rev. Saúde Pública. 1995:29(5):359-63.

8. Oliveira-Filho AD, Morisky DE, Neves SJ, Costa FA, Junior DPL. The 8-item Morisky Medication Adherence Scale: validation of a Brazilian-Portuguese version in hypertensive adults. Research in Social and Administrative Pharmacy. 2014;10(3):554-61. https://doi.org/10.1016/j. sapharm.2013.10.006.

9. Associação Brasileira de Empresas de Pesquisa. Critério de classificação econômica. Brasil: Abep; 2008. [citado 5 mar 2019]. Disponível em: http://www.abep. org.

10. Field A. Descobrindo a estatística usando o SPSS-2. Porto Alegre: Bookman; 2009.

11. Grangeiro A, Escuder MM, Cassanote AJF, Souza RA, Kalichman AO, Veloso V et al. The HIV-Brazil cohort study: design, methods and participant characteristics. PLoS One. 2014;9(5):e95673. https://doi.org/10.1371/journal.pone.0095673.

12. Oliveira FBM, Moura MEB, Araújo TME, Andrade EMLR. Qualidade de vida e fatores associados em pessoas vivendo com HIV/aids. Acta paul. enferm. 2015;28(6):510-6. http://dx.doi. org/10.1590/1982-0194201500086.

13. Brasil. Ministério da Saúde, Secretaria de Vigilância em Saúde. Boletim epidemiológico de aids e DST. Brasília; 2018. [citado 3 fev 2019]. Disponível em: http://www.aids.gov.br/pt-br/ pub/2018/boletim-epidemiologico-hivaids-2018.

14. Minas Gerais. Secretaria Estadual de Saúde, Diretoria de Vigilância Epidemiológica, Coordenação de DST, Aids e Hepatites Virais. Boletim epidemiológico mineiro: análise epidemiológica de HIV/aids. Panorama do ano de 2017. Belo Horizonte; 2018. [citado 5 fev 2019]. Disponível em: http://www.saude.mg.gov.br/images/noticias_e_eventos/000_2019/jane_ fev_mar/BEM\%20Mineiro\%202018HIV.pdf.

15. Bakiono F, Guiguimdé PW, Sanou M, Ouédraogo L, Robert A. Quality of life in persons living with HIV in Burkina Faso: a follow-up over 12 months. BMC Public Health. 2015; 15(1119). http://dx.doi.org/10.1186/s12889-015-2444-4.

16. Liu C, Weber K, Robison E, Hu Z, Jacobson LP, Gange SJ. Assessing the effect of HAART on change in quality of life among HIV-infected women. AIDS Research and Therapy. 2006;3(6):111. http://dx.doi.org/10.1186/1742-6405-3-6. 
17. Solomon S, Batavia A, Venkatesh KK, Brown L, Verma P, Cecelia AJ, Daly C, Mahendra VS, Kumarasamy N, Mayer KH. A longitudinal quality-of-life study of HIV-infected persons in South India: the case for comprehensive clinical care and support services. AIDS Education and Prevention. 2009;21(2):104-12. http://dx.doi.org/10.1521/aeap.2009.21.2.104.

18. Greco DB, Pedroso ERP, Westin MR. Síndrome da imunodeficiência adquirida. In: Pedroso ERP. Série medicina interna: doenças infecciosas. Rio de Janeiro: Rubio; 2015. p. 324-73.

19. Dutra BS, Lédo AP, Lins-Kusterer L, Luz E, Prieto IR, Brites C. Changes health-related quality of life in HIV-infected patients following initiation of antiretroviral therapy: a longitudinal study. Braz J Infect Dis. 2019;23(4):211-7. https://doi.org/10.1016/j.bjid.2019.06.005.

20. Gruszczyńska E, Rzeszutek M. Trajectories of health-related quality of life and perceived social support among people living with HIV undergoing antiretroviral treatment: does gender matter? Front Psychol. 2019;23(10):1664. https://doi.org/10.3389/fpsyg.2019.01664.

21. Medeiros B, Saldanha AAW. Religiosidade e qualidade de vida em pessoas com HIV. Estud. psicol. 2012;29(1):53-61. https://dx.doi.org/10.1590/S0103-166X2012000100006.

22. Razera F, Ferreira J, Bonamigo RR. Factors associated with health-related quality-of-life in HIVinfected Brazilians. International Journal of STD \& AIDS. 2008;19(8):519-23. https://dx.doi. org/10.1258/ijsa.2008.007289.

23. Baral R, Thapa $U$, Khatiwada D. Quality of life among people living with human immunodeficiency virus and acquired immune deficiency syndrome in an anti-retroviral therapy clinic. J Nepal Health Res Counc. 2019;16(41):405-9.

24. Tostes MA, Chalub M, Botega NJ. The quality of life of HIV-infected women is associated with psychiatric morbidity. AIDS Care. 2004;16(2):177-86. https://dx.doi.org/10.1080/095401204100 01641020.

25. Souza Junior PRB, Szwarcwald CL, Castilho EA. Self-rated health by HIV-infected individuals undergoing antiretroviral therapy in Brazil. Cad. Saúde Pública. 2011;27(Suppl 1):s56-s66. http://dx.doi.org/10.1590/S0102-311X2011001300007.

26. Reis AC, Lencastre L, Guerra MP, Remor E. Relação entre sintomatologia psicopatológica, adesão ao tratamento e qualidade de vida na infecção HIV e AIDS. Psicol. Reflex. Crit. 2010;23(3):420-9. https://dx.doi.org/10.1590/S0102-79722010000300002.

27. Leserman J. Role of depression, stress, and trauma in HIV disease progression. Psychosom Med. 2008;70(5):539-45. https://dx.doi.org/10.1097/PSY.0b013e3181777a5f.

28. Yang Y, Thai S, Choi J. An evaluation of quality of life among Cambodian adults living with HIV/ AIDS and using antiretroviral therapy: a short report. AIDS Care. 2016;28(12):1546-50. https:// doi.org/10.1080/09540121.2016.1192100.

29. Campos LN, Cesar CC, Guimarães MD. Quality of life among HIV-infected patients in Brazil after initiation of treatment. Clinics. 2009;64(9):867-75. https://dx.doi.org/10.1590/S180759322009000900007.

Financiamento: O estudo foi financiado pela Fundação de Amparo à Pesquisa do Estado de Minas Gerais (CDS - APQ-03938-16).JOC e JMC receberam bolsa de doutorado da Coordenação de Aperfeiçoamento de Pessoal de Nível Superior -Brasil (CAPES), código de financiamento 001.

Contribuição dos Autores: Concepção e planejamento do estudo: GSP, MRS, MGBC, JOC, JCM, PFB. Coleta de dados: GSP, JOC, JCM. Análise e interpretação dos dados: GSP, JOC, JCM. Todos os autores aprovaram a versão final do manuscrito e assumem a responsabilidade pública pelo seu conteúdo.

Conflito de Interesses: Os autores declaram não haver conflito de interesses. 